\title{
Convergence Theorem for a General Class of Power-Control Algorithms
}

\author{
Kin Kwong Leung, Chi Wan Sung, Member, IEEE, Wing Shing Wong, Fellow, IEEE, and \\ T. M. Lok, Senior Member, IEEE
}

\begin{abstract}
We consider the convergence issues of distributed power-control algorithms for mobile cellular systems. A convergence theorem for power-control algorithms of canonical type is proved. Our result generalizes Yates' framework and provides a new outlook on the problem. The general applicability of the theorem is demonstrated by showing that many well-known distributed algorithms are canonical. Furthermore, by devising some new discrete algorithms, we exemplify how the theorem can be used to aid new design.
\end{abstract}

Index Terms-Canonical algorithm, distributed algorithms, framework, power control.

\section{INTRODUCTION}

$\mathbf{P}$ OWER CONTROL plays an important role in the design of mobile cellular systems. The objective is to manage mutual interference so that every user can have acceptable link quality. There are many ways to formulate the problem, e.g., Zander considered the problem of finding the power vector that maximized the minimum signal-to-interference ratio (SIR) in the system [19]; this formulation is called SIR balancing. Further work proposed distributed balancing algorithms [5], [14], [20]; the performance in fading channels is investigated in [15].

Alongside SIR balancing, another paradigm was established in the literature. For instance, Foschini and Miljanic considered a more general model in which a positive receiver noise and a target SIR were considered [3]. A distributed algorithm was proposed and was shown to converge in both synchronous [3] and asynchronous [10] systems. Based on Foschini and Miljanic's algorithm, Grandhi and Zander suggest a distributed constrained power-control (DCPC) algorithm [6], in which a ceiling is imposed on the transmit power of each user. Another distributed algorithm was proposed by Bambos et al. [1], which aims to protect active links from quality degradation when new users try

Paper approved by D. I. Kim, the Editor for Spread-Spectrum Transmission and Access of the IEEE Communications Society. Manuscript received November 26, 2002; revised August 4, 2003 and December 22, 2003. This work was supported in part by a direct grant from CUHK, in part by the Research Grants Council of the HKSAR under Project CUHK4217/03E, and in part by the City University of Hong Kong under Project 7001454 and Project 7001554. This paper was presented in part at the IEEE International Conference on Communications, Helsinki, Finland, June 2001.

K. K. Leung, W. S. Wong, and T. M. Lok are with the Department of Information Engineering, The Chinese University of Hong Kong, Shatin, NT, Hong Kong (e-mail: kkleung@ie.cuhk.edu.hk; tmlok@ie.cuhk.edu.hk; wswong@ie.cuhk.edu.hk).

C. W. Sung is with the Department of Computer Engineering and Information Technology, City University of Hong Kong, Kowloon, Hong Kong (e-mail: itcwsung@ cityu.edu.hk).

Digital Object Identifier 10.1109/TCOMM.2004.833140 to access the channel. Due to the distributive nature of these algorithms, the most important criterion to be met is the stability, or convergence. In [18], Yates provides a framework for the convergence of power-control algorithms, which may be applied to [1], [3], [6], and [10]. Algorithms that fit into this framework are termed standard algorithms.

In the aforementioned papers, the power levels are allowed to take any positive real values. However, in practical systems, power levels are quantized. Based on this observation, Sung and Wong proposed the fixed-step algorithm (FS algorithm) [13]. Since this new algorithm is not standard, a convergence proof is developed in [13]. The convergence proof of [13] hints at the possibility that Yates' framework may be further generalized.

The essence of Yates' framework is the generalization of the interference measure. It is shown that for a broad class of powercontrolled systems, the interference measure satisfies the positivity, monotonicity, and scalability conditions. Coincidently, it is shown in [12] that the FS algorithm also converges under the same type of interference measures; this motivates us to explore the reasons for this similarity.

In this paper, we establish a more general framework on convergence analysis. We identify two crucial conditions for the convergence of power-control algorithms. Algorithms that satisfy these conditions are called canonical. We show that standard algorithms form a proper subset of the canonical ones, which implies that our new framework can be applied to a broader class of algorithms. In fact, most well-known distributed algorithms fit into our framework; the only exception is the second-order power control in [8]. Two new algorithms, developed in this paper, demonstrate that our result may provide guidelines for the design of more sophisticated power-control algorithms.

The rest of the paper is organized as follows. In Section II, we describe the system model and define some terms. In Section III, we have a review of the fixed-step power-control algorithm, which is used to illustrate the concepts of the canonical algorithm in Section IV. In Section IV, we state and investigate the canonical algorithm. In Section V, we give applications of the canonical convergence theorems. The paper is concluded in Section VI.

\section{SYSTEM MODEL}

Consider a cellular system, with $N$ mobile units assigned to a set of base stations. Let $\boldsymbol{P}=\left(P_{1}, P_{2}, \ldots, P_{N}\right)$, where $P_{i} \in \overline{\mathbb{R}}_{+}$is the power transmitted by the $i$ th mobile. Here $\overline{\mathbb{R}}_{+}$represents the set of all nonnegative real numbers. We use 
$\Gamma_{i}(\boldsymbol{P})$ to represent the quality of service $(\mathrm{QoS})$ of the $i$ th mobile, and $\boldsymbol{\Gamma}(\mathbf{P})=\left(\Gamma_{1}(\boldsymbol{P}), \Gamma_{2}(\boldsymbol{P}), \Gamma_{3}(\boldsymbol{P}), \ldots, \Gamma_{N}(\boldsymbol{P})\right)$. Examples of QoS includes signal-to-noise ratio (SNR), bit-error rate (BER), and frame-error rate (FER). Clearly, $\boldsymbol{\Gamma}(\boldsymbol{P})$ is dependent on the power of the mobiles $\boldsymbol{P}$. In a real situation, the function $\boldsymbol{\Gamma}(\boldsymbol{P})$ may be time varying, since the locations of the mobiles are changing. However, in our model, the power-control update process is frequent enough that we may assume the channel is static throughout the process.

\section{A. Totally Asynchronous Model}

In this section, we apply the totally asynchronous model in [2] to the power-control problem. In our model, the mobiles are allowed to update their power without a central coordinator, and some mobiles may update their power much more frequently than the others. Moreover, the interference is composed of the power transmitted by the other users, and there may be an unbounded delay on estimating the transmitted power of other users. The definition follows.

Let $P_{i}^{(t)}$ be the power of mobile $i$ at time $t$. Similarly, we have $\Gamma_{i}^{(t)}$ and $I_{i}^{(t)}$ defined. Without loss of generality, we assume that the set of times at which one or more components $P_{i}$ of $\boldsymbol{P}$ are updated is the discrete set $T=\{0,1,2, \ldots\}$. We follow the formulation in [2] that the update is carried out just after $t$. We assume that $\Gamma_{i}^{(t)}$ may not be calculated from the most recent value of $\boldsymbol{P}$. Let $\tau_{j}^{i}(t)$ be the time corresponding to the $j$ th component used in calculating $\Gamma_{i}^{(t)} i$. In other words

$$
\Gamma_{i}^{(t)}=\Gamma_{i}\left(P_{1}^{\left(\tau_{1}^{i}(t)\right)}, P_{2}^{\left(\tau_{2}^{i}(t)\right)}, \ldots, P_{N}^{\left(\tau_{N}^{i}(t)\right)}\right) \quad \forall t \in T
$$

where $\tau_{j}^{i}(t)$ are times satisfying $0 \leq \tau_{j}^{i}(t) \leq t$.

Definition 1: A system is called totally asynchronous if $\lim _{t \rightarrow \infty} \tau_{j}^{i}(t)=\infty$ for every mobile $i, j$.

This assumption guarantees that old information is eventually purged from the system. More precisely, given any time $t_{1}$, there exists a time $t_{2}>t_{1}$ such that $\tau_{j}^{i}(t) \geq t_{1}$, for all $i, j$, and $t \geq t_{2}$.

\section{B. Standard Interference Function}

Recall that $\Gamma_{i}(\boldsymbol{P})$ is a general QoS measure of user $i$. We call $I_{i}(P)$ the interference measure of user $i$ if the following relation holds:

$$
\Gamma_{i}(\boldsymbol{P})=\frac{P_{i}}{I_{i}(\boldsymbol{P})} .
$$

In fact, for $P_{i}>0, I_{i}(\boldsymbol{P})$ may be defined as

$$
\frac{P_{i}}{\Gamma_{i}(\boldsymbol{P})} .
$$

Definition 2: An interference measure $I_{i}(\boldsymbol{P})$ is called standard if it satisfies the following two conditions.

1) Scalability: For all $\alpha>1, \alpha \boldsymbol{I}(\boldsymbol{P})-\boldsymbol{I}(\alpha \boldsymbol{P})>0$.

2) Monotonicity: If $\boldsymbol{P} \geq \boldsymbol{P}^{\prime}$, then $\boldsymbol{I}(\boldsymbol{P}) \geq \boldsymbol{I}\left(\boldsymbol{P}^{\prime}\right)$.

The idea of standard interference function comes from [18], where it was coupled with the standard power-control algorithm. In this paper, we generalize the approach of [12] by employing the standard interference function to prove the convergence of algorithms outside the standard power-control framework of [18].
In the original definition of standard interference function, there is one more condition - the positivity condition: $I_{i}(\boldsymbol{P})>$ 0 . However, it is just a consequence of the other two conditions, as the following lemma shows.

Lemma 1: Positivity: $I(P)>0$.

Proof: Since $\boldsymbol{P} \geq 0$, by scalability and monotonicity, we have $2 \boldsymbol{I}(\boldsymbol{P})>\boldsymbol{I}(2 \boldsymbol{P}) \geq \boldsymbol{I}(\boldsymbol{P})$.

Some more properties of the standard interference function $I_{i}(\boldsymbol{P})$ follow; these properties are essential in proving the convergence theorem.

Lemma 2: $\boldsymbol{I}(\boldsymbol{P})$ is continuous.

The proof of Lemma 2 can be found in [7].

Scalability states that $\alpha \boldsymbol{I}(\boldsymbol{P})-\boldsymbol{I}(\alpha \boldsymbol{P})>0$ for any $\alpha>1$. In Lemma 3, we prove that the expression is lower bounded by a positive real number independent of $\boldsymbol{P}$, if $\boldsymbol{P}$ is bounded. In other words, the scalability is uniform, as defined below.

Definition 3: $\boldsymbol{f}(\boldsymbol{x})$ is called uniformly scalable in $X$ if given any $\alpha>1$, there is $\epsilon(\alpha)>0$ such that

$$
\alpha \boldsymbol{f}(\boldsymbol{x})-\boldsymbol{f}(\alpha \boldsymbol{x})>\boldsymbol{\epsilon}(\alpha) \quad \forall \boldsymbol{x} \in X .
$$

Lemma 3: $\boldsymbol{I}(\boldsymbol{P})$ is uniformly scalable in $\mathcal{P}$, where $\mathcal{P}=\mathcal{P}_{1} \times$ $\mathcal{P}_{2} \times \mathcal{P}_{3} \times \cdots \times \mathcal{P}_{N}$, and $\mathcal{P}_{i}=\left[0, p_{i}\right]$

Proof: Let $\boldsymbol{h}_{\alpha}(\boldsymbol{P})=\alpha \boldsymbol{I}(\boldsymbol{P})-\boldsymbol{I}(\alpha \boldsymbol{P})$. By the scalability of $\boldsymbol{I}(\boldsymbol{P}), \boldsymbol{h}_{\alpha}(\boldsymbol{P})>0$ for any $\alpha>1$. Since $\boldsymbol{I}(\boldsymbol{P})$ is continuous, $\boldsymbol{h}_{\alpha}(\boldsymbol{P})$ is also continuous. Together with the fact that $\mathcal{P}$ is compact [11], the image $\boldsymbol{h}_{\alpha}(\mathcal{P})$ is also a compact subset in $\overline{\mathbb{R}}_{+}$. Hence, a positive minimum $\epsilon_{i}(\alpha)$ exists in $\boldsymbol{h}_{\alpha}(\mathcal{P})$. We have $\boldsymbol{h}_{\alpha}(\boldsymbol{P}) \geq \epsilon_{i}(\alpha)$.

\section{Some ObServations Regarding the FS Algorithm}

In this section, we have a brief review of the FS algorithm proposed in [13]. It will be used as example to illustrate the concepts behind the canonical algorithm in the next section. The FS algorithm may be stated as follows: ${ }^{1}$

$$
P_{i}^{(t+1)}= \begin{cases}\delta P_{i}^{(t)}, & \text { if } \Gamma_{i}^{(t)}<\delta^{-1} \gamma_{F} \\ \delta^{-1} P_{i}^{(t)}, & \text { if } \Gamma_{i}^{(t)}>\delta \gamma_{F} \\ P_{i}^{(t)}, & \text { otherwise }\end{cases}
$$

where $\delta>1 . \gamma_{F}$ is the target SIR of the algorithm.

The first observation is on the convergent region of the algorithm. Although $\gamma_{F}$ is called the target SIR, it plays no important role in the power-control operation. It is only used as a means to specify the lower and the upper threshold of the convergent region. One can generalize the algorithm by defining the convergent region as $[l, u]$. If the SIR of a user is above this region, its power is decreased by $\delta$; if the SIR is below the region, the power is increased by $\delta$. What really matters is the width of the region. It is intuitively clear that the algorithm converges only if the region is wide enough.

The second observation regards the iterations of the algorithm. In the case of $\Gamma_{i}^{(t)}<\delta^{-1} \gamma_{F}, P_{i}^{(t)}$ is increased in a way such that the resultant $\Gamma_{i}$ may never exceed $\gamma_{F}$, given that the interference is kept constant, i.e.,

$$
P_{i}^{(t+1)} / I_{i}^{(t)} \leq \gamma_{F}
$$

${ }^{1}$ For simplicity, all users are assumed to have the same target SIR, $\gamma_{F}$. In the original algorithm, which is also described in Section V-C, different users may have different target SIR. 
hence

$$
P_{i}^{(t)} \leq P_{i}^{(t+1)} \leq \gamma_{F} I_{i}^{(t)}
$$

Similarly, in the case of $\Gamma_{i}^{(t)}>\delta \gamma_{F}$, we find

$$
\gamma_{F} I_{i}^{(t)} \leq P_{i}^{(t+1)} \leq P_{i}^{(t)}
$$

Combining (2) and (3), the following relationship is found:

$$
\min \left(P_{i}^{(t)}, \gamma_{F} I_{i}^{(t)}\right) \leq P_{i}^{(t+1)} \leq \max \left(P_{i}^{(t)}, \gamma_{F} I_{i}^{(t)}\right)
$$

Now consider the generalized algorithm in which the convergent region is specified by $[l, u]$. We can always find $\gamma_{F} \in[l, u]$ such that (4) holds, provided that $u / l \geq \delta^{2}$. This is a key property for the algorithm to converge, as will be seen in the next section.

Following these observations, we are able to introduce the canonical algorithm.

\section{CAnOnical Algorithm}

In this section, we introduce the canonical algorithm. A power-control algorithm is an algorithm which updates the power vector from $\boldsymbol{P}^{(t)}$ to $\boldsymbol{P}^{(t+1)}$. The first condition for a power-control algorithm to be canonical is that the interference measure is standard. Apart from this, the concept of the canonical power-control algorithm is composed of two other parts, the target region and the power update.

\section{A. Target Region}

Let $\mathcal{T}_{i}$ be a subset of the set of all positive real numbers, $\mathbb{R}_{+}$. When $\Gamma_{i} \in \mathcal{T}_{i}$, the link quality of mobile $i$ is regarded as acceptable. The objective of power control is to find a power vector $\boldsymbol{P}$ such that $\Gamma_{i}(\boldsymbol{P}) \in \mathcal{T}_{i}$ for all $i$, where $\mathcal{T}_{i}$ is the target region of mobile $i$. The system target region is defined as $\mathcal{T}=$ $\mathcal{T}_{1} \times \mathcal{T}_{2} \times \mathcal{T}_{3} \times \cdots \times \mathcal{T}_{N}$.

The corresponding component of the target region in the FS algorithm is the convergent region $\left[\delta^{-1} \gamma_{F}, \delta \gamma_{F}\right]$, while the system target region is the $N$-dimensional product space $\prod_{i=1}^{N}\left[\delta^{-1} \gamma_{F}, \delta \gamma_{F}\right]$

\section{B. Update Algorithm}

The change of power level from $P_{i}^{(t)}$ to $P_{i}^{(t+1)}$ is called a power update. To ensure the convergence of the power vector, there are two essential conditions on the update algorithms. Before defining these conditions, we have to introduce the concept of separation between two sets, and the feasibility of a QoS vector. We define separation using concepts in topology [11].

Definition 4: Let $X, Y \subseteq \mathbb{R}^{n}, \bar{X}$ be the closure of $X$ and $\bar{Y}$ be the closure of $Y$. We say that $X$ is separated from $Y$ if $X \cap \bar{Y}=Y \cap \bar{X}=\phi$.

In other words, two sets are separated if they are disjoint and do not touch each other. For example, $(0,1)$ and $(1,2)$ are separated sets. However, $(0,1]$ and $(1,2)$ are not separated.

Definition 5: A QoS vector $\gamma \in \overline{\mathbb{R}}_{+}^{N}$ is called feasible if there exists a power vector $\boldsymbol{P} \in \overline{\mathbb{R}}_{+}^{N}$ such that

$$
\Gamma(P)=\gamma \text {. }
$$

Now we are ready to introduce the bounding condition and the reactive condition.

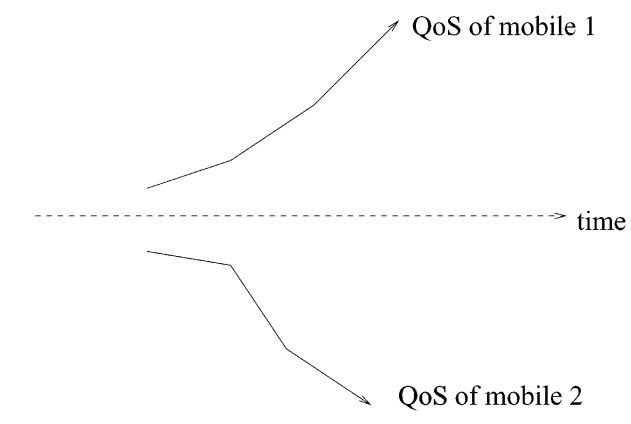

Fig. 1. QoS of the two mobiles changes in the direction opposite to the target QoS.

Definition 6: An update algorithm is called bounded if it satisfies the bounding condition; it is called reactive if it satisfies the reactive condition.

1) Bounding Condition: There exists a feasible $\boldsymbol{\gamma}=$ $\left(\gamma_{1}, \gamma_{2}, \gamma_{3}, \ldots, \gamma_{N}\right) \in \mathcal{T}$ such that

$\min \left(P_{i}^{(t)}, \gamma_{i} I_{i}^{(t)}\right) \leq P_{i}^{(t+1)} \leq \max \left(P_{i}^{(t)}, \gamma_{i} I_{i}^{(t)}\right)$

for any $i$ and $t$. We call $\gamma$ the target QoS vector and $\gamma_{i}$ the target QoS of mobile $i$.

2) Reactive Condition: For any mobile $i$ and $X \subseteq \overline{\mathbb{R}}_{+}$where $X$ is separated from the target region $\mathcal{T}_{i}$, there exists $\epsilon>0$ and an infinite subset of nonnegative integers $T^{i}$, such that

$$
\left|\frac{P_{i}^{(t)}}{P_{i}^{(t+1)}}-1\right|>\epsilon
$$

whenever $\Gamma_{i}^{(t)} \in X$ and $t \in T^{i}$.

$P_{i}^{(t)}$ and $\gamma_{i} I_{i}^{(t)}$ in the bounding condition forms a lower and an upper bound on the power level at the next update. Consider the case of $P_{i}^{(t)} \leq \gamma_{i} I_{i}^{(t)}$ (power not high enough). If $P_{i}^{(t+1)}$ is set below $P_{i}^{(t)}$ (decrease in power), the QoS will move away from the target QoS, $\gamma_{i}$, as mobile 2 shown in Fig. 1. Alternatively, if $P_{i}^{(t+1)}$ is set above $\gamma_{i} I_{i}^{(t)}$, the resultant QoS will overshoot the target. This overshoot in a multiuser system may cause the QoS to oscillate, and eventually move further and further from the target, as shown in Fig. 2.

Under the reactive condition, the QoS of the mobiles are not allowed to stay outside the target region indefinitely. The power significantly changes when the target region is not reached.

Roughly stated, if these conditions are satisfied, the power of each mobile moves toward the target, and it will not stop moving until the target is reached. In addition, with the condition that the target QoS vector is feasible, which is included in the bounding condition, we can prove that the QoS vector converges to the system target region. The results are formally stated in the next subsection.

\section{Power-Control Theorems}

Definition 7: A system is called weakly canonical if the update algorithms of all users are bounded and their interference measures are standard.

Theorem 4 (Bounded Power Theorem): In a weakly canonical system, the power vector $\boldsymbol{P}^{(t)}$ is bounded for any initial power vector. 


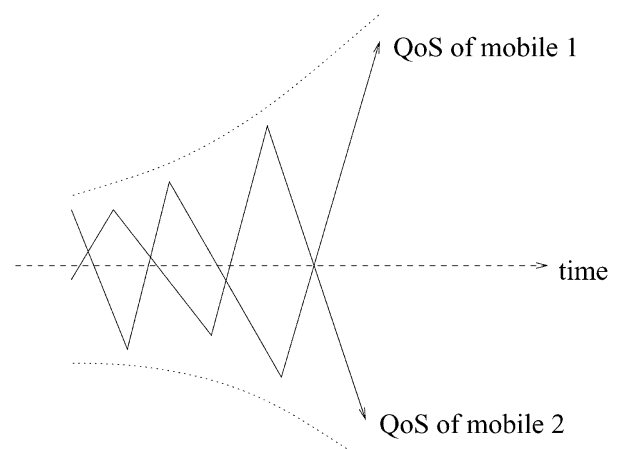

Fig. 2. QoS of the mobiles changes in the correct direction but with too large a step, and hence moves further and further away from the target QoS.

Proof: Let $\boldsymbol{\gamma}$ be a target QoS, and $\boldsymbol{P}^{*}=$ $\left(P_{1}^{*}, P_{2}^{*}, P_{3}^{*}, \ldots, P_{N}^{*}\right)$ be the corresponding power solution, i.e., $\boldsymbol{\Gamma}\left(\boldsymbol{P}^{*}\right)=\gamma$. Rewrite $\left(P_{1}^{(t)}, P_{2}^{(t)}, P_{3}^{(t)}, \ldots, P_{N}^{(t)}\right)$ as $\left(P_{1}^{*} \mu_{1}^{(t)}, P_{2}^{*} \mu_{2}^{(t)}, P_{3}^{*} \mu_{3}^{(t)}, \ldots, P_{N}^{*} \mu_{N}^{(t)}\right)$. Let $\mu_{0}=\max _{i}$ $\left\{\mu_{i}^{(0)}, 1\right\}$. We now prove that $\mu_{i}^{(t)} \leq \mu_{0}$ for any $i, t$ by mathematical induction in $t$.

It is true for $t=0$ by definition. Assume that it is true for all $t<T$. Consider mobile $i$ for the following two cases.

1) If $i$ does not increase its power at time $T, \mu_{i}^{(T)} \leq \mu_{0}$.

2) If $i$ increases its power at time $T$, then by the bounding condition, $P_{i}^{(T-1)}<P_{i}^{(T)} \leq \gamma_{i} I_{i}^{(T-1)}$. Hence

$$
\begin{aligned}
P_{i}^{(T)} & \leq \gamma_{i} I_{i}^{(T-1)} \\
P_{i}^{*} \mu_{i}^{(T)} & \leq \gamma_{i} I_{i}^{(T-1)} \\
P_{i}^{*} \mu_{i}^{(T)} & \leq \gamma_{i} I_{i}\left(P_{1}^{*} \mu_{1}^{\left(\tau_{i}^{1}(T-1)\right)}, \ldots, P_{2}^{*} \mu_{N}^{\left(\tau_{i}^{N}(T-1)\right)}\right) \\
& \leq \gamma_{i} I_{i}\left(\mu_{0} \boldsymbol{P}^{*}\right) \\
& \leq \gamma_{i} \mu_{0} I_{i}\left(\boldsymbol{P}^{*}\right) \\
\frac{P_{i}^{*}}{I_{i}\left(\boldsymbol{P}^{*}\right)} \mu_{i}^{(T)} & \leq \gamma_{i} \mu_{0} \\
\mu_{i}^{(T)} & \leq \mu_{0}
\end{aligned}
$$

where (5), (6), and (7) follow from the monotonicity, scalability, and positivity of $I_{i}(\boldsymbol{P})$, respectively.

Hence, for any mobile $i, \mu_{i}^{(T)} \leq \mu_{0}$ in both cases and the induction follows. It means that $P^{(t)}$ is bounded above. On the other hand, the power is bounded below by 0 , because $P_{i}^{(T)}$ must be between $P_{i}^{(T-1)}$ and $\gamma_{i} I_{i}^{(T)}$, which are nonnegative.

The bounded power theorem is essential in proving the other theorem; besides, it implies that the algorithm is stable in some sense.

Theorem 5 (Convergent Power-Control Theorem): In a weakly canonical system, $\lim _{t \rightarrow \infty} \boldsymbol{P}^{(t)}$ and $\lim _{t \rightarrow \infty} \Gamma\left(\boldsymbol{P}^{(t)}\right)$ exist.

Due to the length of the proof for Theorem 5, it is placed in the Appendix.

Definition 8: The power-control algorithm of user $i$ is called canonical if the following are satisfied:

1) the whole system is weakly canonical;

2) the update algorithm of user $i$ is reactive;

3) the target region $\mathcal{T}_{i}$ is closed.
If the power-control algorithms of all users in a system is canonical, we call it a canonical system. In other words, the powercontrol algorithms of the users in a canonical system satisfy the following:

1) the interference measure is standard;

2) the target region is closed;

3) the update algorithm is bounded and reactive.

Theorem 6 (Canonical Power-Control Theorem): If the power-control algorithm of user $i$ is canonical, then

$$
\lim _{t \rightarrow \infty} \Gamma_{i}\left(\boldsymbol{P}^{(t)}\right) \in \mathcal{T}_{i}
$$

Proof: Since the system is weakly canonical, by Theorem $5, \lim _{t \rightarrow \infty} \boldsymbol{\Gamma}_{i}\left(\boldsymbol{P}^{(t)}\right)$ exists. Moreover, given that the update algorithm is reactive, and that $\lim _{t \rightarrow \infty} \boldsymbol{P}^{(t)}$ exists with $\mathcal{T}_{i}$ closed, then $\lim _{t \rightarrow \infty} \Gamma_{i}\left(\boldsymbol{P}^{(t)}\right) \in \mathcal{T}_{i}$.

This theorem guarantees that a canonical algorithm always converges, and the resulting QoS vector falls within the target region. In Yates' framework, the limit point is unique; it corresponds to the special case where $\mathcal{T}$ consists of only one single point. In general, multiple limit points exist. The limit point depends on the initial vector, the power-update time instants, the delay, and the actual power-update algorithms. For example, the limit point of the FS algorithm under synchronous update depends on the initial vector.

Another remark is that in Yates' framework, a condition is required for the convergence: a feasible power vector exists. This condition is also needed in our framework. However, it is implicitly stated in the bounding condition. In other words, if such a vector does not exist, the bounding condition cannot be satisfied.

\section{Maximum Power Constraints and Multiple Limit Points}

In practice, the transmit power is usually constrained to a maximum level. In Yates' framework, this problem is handled by modifying the interference function. Our approach provides another viewpoint. Let $M_{i}$ be the maximum power of user $i$. Suppose the power of user $i$ is updated from $P_{i}^{(t)}$ to $P_{i}^{(t+1)}$ according to a certain algorithm. If $P_{i}^{(t+1)}$ exceeds $M_{i}, P_{i}^{(t+1)}$ is forced to be $M_{i}$. It is easy to show that a bounded power-update algorithm with this constrained operation is still bounded. Thus, the power and the QoS vectors converge; let their limits be $\boldsymbol{P}^{*}$ and $\boldsymbol{\Gamma}^{*}$. However, there is no guarantee that every $\Gamma_{i}^{*}$ is a member of the target region $\mathcal{T}_{i}$, since the reactive condition may be violated when $P_{i}^{*}=M_{i}$. If $P_{i}^{*}<M_{i}$, then $\Gamma_{i}^{*}$ belongs to $\mathcal{T}_{i}$. Note that with power contraints, the existence of a feasible power vector within the constrained set does not imply that the limit point, $\Gamma^{*}$, will fall within $\mathcal{T}$, unless $\mathcal{T}$ consists of only a single point as in Yates' framework. An example follows.

Consider a two-user system with QoS measure $\boldsymbol{\Gamma}(\boldsymbol{P})=$ $\left(\left(P_{1}\right) /\left(P_{2}+1\right),\left(P_{2}\right) /\left(P_{1}+1\right)\right)$. Let the target region of both users be the interval $[1 / 2,10]$, and the power constraints $M_{1}=1$ and $M_{2}=10$. Obviously, the power vector $\boldsymbol{P}=(1,1)$ satisfies the power contraints and the QoS requirements. Therefore, feasible power vector exists in the system. However, $\boldsymbol{P}^{(0)}=(1,10)$ may result in $\boldsymbol{P}^{*}=(1,10)$, where the corresponding $\Gamma_{1}^{*}=1 / 11$ does not fall into $\mathcal{T}_{1}$. 


\section{APPLiCATIONS OF THE THEOREM}

The canonical convergence theorem applies to a broad range of power-control algorithms. In this section, we examine a few examples; the convergence of some of them is proved for the first time. Furthermore, the theorem provides a guideline for the design of new algorithms; we demonstrate this by developing the algorithms in Sections V-D and V-E.

\section{A. Foschini and Miljanic's Standard Algorithm}

The algorithm proposed by Foschini and Miljanic updates the power of the mobiles as follows [3]:

$$
P_{i}^{(t+1)}=\gamma_{i} I_{i}^{(t)}
$$

where $I_{i}^{(t)}$ is of the form $I_{i}^{(t)}=\sum_{j \neq i} G_{i j} P_{j}+\eta_{i}$ and $\gamma_{i}$ is the target QoS.

This algorithm was proved to converge when all users update their power simultaneously. This result was later extended to a totally asynchronous system by Mitra [10]. Yates further generalized the result such that the algorithm converges under any standard interference measure [18]. All these results may be obtained by the canonical convergence theorem. We prove the algorithm is canonical by checking the following properties.

1) Interference Measure: The interference measure is standard.

2) Target Region: The target region $\mathcal{T}_{i}$ is $\left[\gamma_{i}, \gamma_{i}\right]$, which is closed in $\mathbb{R}_{+}$.

3) Bounding Condition: The update $P_{i}^{(t+1)}=\gamma_{i} I_{i}^{(t)}$ satisfies the bounding condition with the target QoS $\gamma_{i}$.

4) Reactive Condition: Let $A$ be a set in $\overline{\mathbb{R}}_{+}$separated from $\mathcal{T}_{i}$. Then there exists $\epsilon>0$ such that $\left[(1 / 1+\epsilon) \gamma_{i},(1 / 1-\right.$ $\left.\epsilon) \gamma_{i}\right]$ is disjoint from $A$. For any $t \in T^{i}$, if $\Gamma_{i} \in A$, then $\left|\left(P_{i}^{(t)}\right) /\left(\gamma_{i} I_{i}^{(t)}\right)-1\right|>\epsilon$. Also, $P_{i}^{(t+1)}=\gamma_{i} I_{i}^{(t)}$. Hence, we have

$$
\left|\frac{P_{i}^{(t)}}{P_{i}^{(t+1)}}-1\right|>\epsilon
$$

Hence, standard algorithm is canonical and its convergence is implied by the canonical power-control theorem.

\section{B. Bambos et al.'s ALP Algorithm}

The standard algorithm allows the link QoS to drop below the target during the evolution. Therefore, when new links try to access the channel, established links may inadvertently be dropped due to a temporary QoS degradation below an acceptable levels. To prevent this, Bambos et al. proposed an active-link-protection (ALP) algorithm [1]. The iterative procedure is as follows:

$$
P_{i}^{(t+1)}= \begin{cases}\delta \gamma_{i} I_{i}^{(t)}, & \text { if } \Gamma_{i}^{(t)} \geq \gamma_{i} \\ \delta P_{i}^{(t)}, & \text { if } \Gamma_{i}^{(t)}<\gamma_{i}\end{cases}
$$

The interference measure is assumed to be standard and it is easy to check that the update algorithm is bounded. Therefore, the system is weakly canonical. The convergence is implied by the convergent power-control theorem. For those with nonzero initial power, the reactive condition is also satisfied. Their power- control algorithm is hence canonical, with target region $\mathcal{T}_{i}=$ $\left\{\delta \gamma_{i}\right\} . \Gamma_{i}$ is guaranteed to converge to $\delta \gamma_{i}$.

\section{Sung and Wong's FS Algorithm}

As discussed in previous sections, the update algorithm of the FS algorithm satisfies the bounding condition and the target region $\mathcal{T}_{i}=\left[\delta^{-1} \gamma_{F}, \delta \gamma_{F}\right]$ is closed. If we assume that all users have nonzero initial power, the reactive condition is also satisfied and the algorithm is proved to converge under standard interference measures.

\section{Asymmetric Step Algorithm}

Since power control cannot be perfect, there is nonzero probability that the SIR of a mobile falls below the acceptable level (outage probability). A common method to reduce the outage probability is to use excessive power in the transmission. The amount of excesssive power is called the fade margin. In [9], we propose the asymmetric step power-control algorithm, which can save up to $3 \mathrm{~dB}$ of power to achieve the same outage probability, when compared with the fixed-step power-control algorithm. The power is adjusted as follows.

For each mobile $i$

$$
P_{i}^{(t+1)}= \begin{cases}\alpha_{i} P_{i}^{(t)}, & \text { if } \Gamma_{i}^{(t)}<\text { lower threshold } \\ \beta_{i}^{-1} P_{i}^{(t)}, & \text { if } \Gamma_{i}^{(t)}>\text { upper threshold } \\ P_{i}^{(t)}, & \text { otherwise }\end{cases}
$$

where $\alpha_{i}, \beta_{i}>1$.

According to the theorem, the target region should be $\left[\alpha_{i}^{-1} \gamma_{i}, \beta_{i} \gamma_{i}\right]$. In other words, the lower threshold is $\alpha^{-1} \gamma_{i}$ and the upper threshold is $\beta_{i} \gamma_{i}$. Then the algorithm is canonical, and its convergence follows.

Simulations show that different incremental step sizes $\alpha_{i}$ give different outage probabilities. In addition, the theorem allows different users to have different $\alpha_{i}$ and $\beta_{i}$ to meet their own service requirements.

\section{E. Discrete Standard Algorithm}

The standard algorithm in Section V-A assumes a continuous-valued power system. In practical systems, there is a finite number of discrete power levels. Let us suppose the power levels are quantized uniformly along a logarithmic scale. In the linear scale, the levels can be represented by $\left\{\ldots, \delta^{-2} p_{0}, \delta^{-1} p_{o}, p_{0}, \delta p_{0}, \delta^{2} p_{o}, \ldots\right\}$, where $\delta>1$ is the quantization step size. A straightforward modification to the standard algorithm may allow every mobile to take the nearest value at each update. However, it can be shown that this modification to the standard algorithm does not guarantee convergence, even in synchronous system. An example follows.

Consider a two-user system with SIR measure $\boldsymbol{\Gamma}\left(P_{1}, P_{2}\right)=$ $\left(\left(P_{1}\right) /\left(P_{2}+1\right),\left(P_{2}\right) /\left(P_{1}+1\right)\right)$. The available power levels are $\{2.25,4.5,9,18,36, \ldots\}$, i.e., $\delta=2 . \gamma=(0.9,0.9)$ is a feasible target, with corresponding power vector $\boldsymbol{P}=(9,9)$. Obviously, $\left(P_{j}+1\right)$ satisfies the two requirements of standard interference measure. If the initial power $\boldsymbol{P}^{(0)}=(2.25,36)$, according to the modified algorithm, $P^{(1)}=(36,2.25)$, because $(33.3,2.925)$ is not an available power vector. Similarly, $\boldsymbol{P}^{(2)}$ will return to $(2.25,36)$. Clearly, $P$ is oscillating and hence, never converges, 


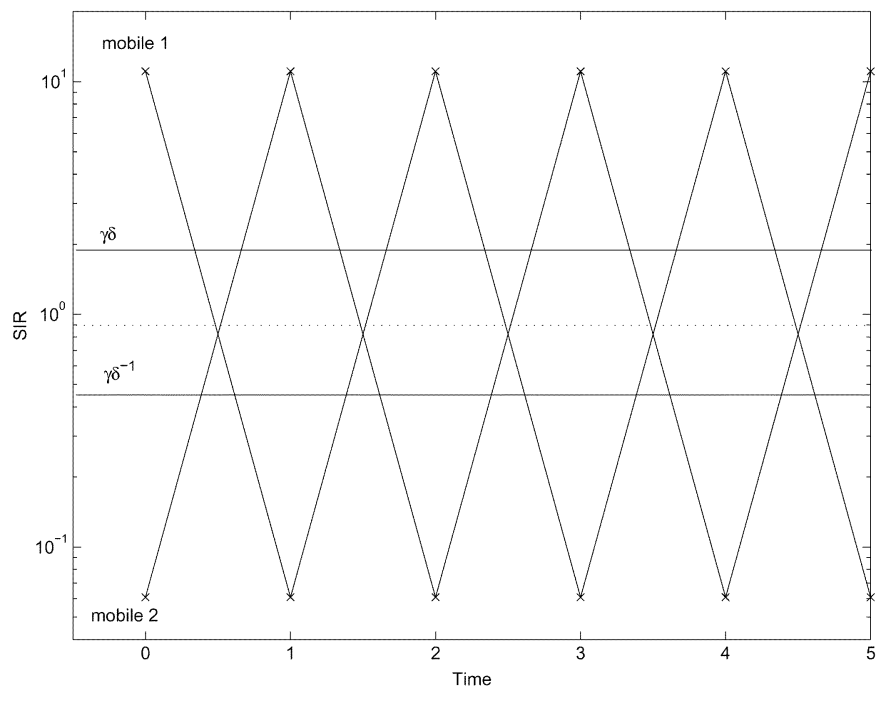

Fig. 3. SIR of the users oscilates when a straightforward modification is applied to the quantized power-control algorithm.

as shown in Fig. 3. The SIR of the users are far from the target region.

The canonical power-control theorem suggests a feasible modification to the standard algorithm. We let $\lfloor x\rfloor$ be the largest quantized power level that is smaller than or equal to $x$. Similarly, we let $\lceil x\rceil$ be the smallest quantized power level that is larger than or equal to $x$. A discrete version of the standard algorithm is

$$
P_{i}^{(t+1)}= \begin{cases}\left\lfloor\gamma_{i} I_{i}^{(t)}\right\rfloor, & \text { if } \Gamma_{i}^{(t)}<\delta^{-1} \gamma_{i} \\ \left\lceil\gamma_{i} I_{i}^{(t)}\right\rceil, & \text { if } \Gamma_{i}^{(t)}>\delta \gamma_{i} \\ P_{i}^{(t)}, & \text { otherwise. }\end{cases}
$$

This discrete algorithm does not fall within Yates' framework [18]. However, it is trivial to show that this algorithm satisfies the bounding condition. Hence, its convergence is guaranteed by the convergent power-control theorem. Further investigation demonstrates that the target region for constructing a canonical power-control algorithm is $\left[\delta^{-1} \gamma_{i}, \delta \gamma_{i}\right]$. The SIR of the two-user system with this modified algorithm is shown in Fig. 4.

Note that in designing this algorithm, the output of the standard algorithm is determined from (11), so that the bounding conditions are satisfied. This example highlights how the theorems may be used to aid the design of new algorithms.

\section{CONCLUSION}

A new framework for distributed power control is established. By identifying two crucial conditions for convergence, the bounding and reactive conditions, we define a canonical form for a class of power-control algorithms. We show that all canonical algorithms converge in a totally asynchronous system. This canonical class covers many well-known algorithms in the literature, some of which had not been shown to converge until now. Through this investigation, we have developed a deeper insight into the design of better power-control algorithms.

As a remark, we note that our framework applies only to the QoS tracking problem. Recently, a new power-control paradigm

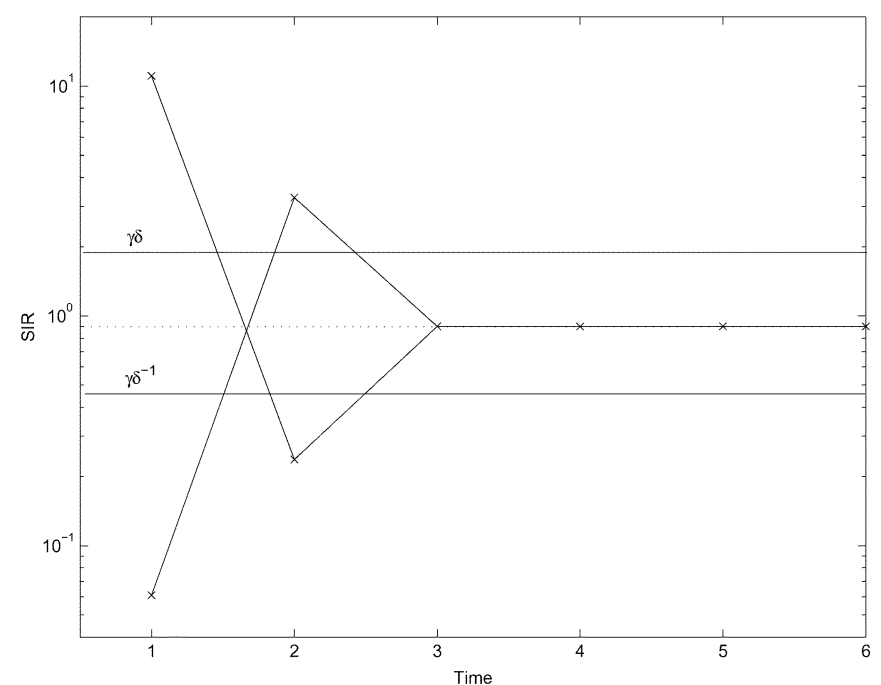

Fig. 4. SIR of the users converges when the quantized power-control algorithm is modified according to the canonical power-control algorithm.

based on game theory emerges [4], [16], [17]. The convergence of algorithms under this paradigm requires further investigation.

\section{APPENDIX \\ PROOF OF THEOREM 5}

In this section, we will prove the convergent power-control theorem. We assume a totally asynchronous system with a standard interference measure. For simplicity, we use $\boldsymbol{P}^{\left(\vec{\tau}_{i}(t)\right)}$ to represent the vector $\left(P_{i}^{\left(\tau_{1}^{i}(t)\right)}, P_{2}^{\left(\tau_{2}^{i}(t)\right)}, \ldots, P_{N}^{\left(\tau_{N}^{i}(t)\right)}\right)$, and we use $I_{i}^{(t)}$ to represent $I_{i}\left(\boldsymbol{P}^{\left(\vec{\tau}_{i}(t)\right)}\right)$.

At any time $t$, we can always divide the mobiles into the following three categories:

1) power of mobiles goes up or down monotonically after time $t$;

2) power of mobiles goes both up and down after time $t$;

3) power of mobiles is unchanged after time $t$.

Define $\mathcal{A}(t), \mathcal{B}(t)$, and $\mathcal{C}(t)$ to be the sets containing the mobiles of categories 1,2 , and 3 at time $t$, respectively. The set $\mathcal{A}(t), \mathcal{B}(t)$, and $\mathcal{C}(t)$ form a partition over the set of all mobiles.

For any scenario, we define $t_{0}, t_{1}, t_{2}, \ldots$ and $t_{0}^{\prime}, t_{1}^{\prime}, t_{2}^{\prime}, \ldots$ as follows. Let $t_{0}$ and $t_{0}^{\prime}$ be time 0 , and define $t_{n+1}^{\prime}=\min \{T$ : $\tau_{j}^{i}(t)>t_{n} \forall i, j$ and $\left.t>T\right\}$. The minimum exists since it is minimized over a subset of positive integers. This $t_{n+1}^{\prime}$ is a point where the values before $t_{n}$ will not be used anymore. In other words, for the power control after $t_{n+1}^{\prime}$, the delayed samples will not be older than $t_{n}$. Since the mobiles in $\mathcal{A}\left(t_{n+1}^{\prime}\right)$ change the power monotonically and their power is bounded, each of their power will approach a limiting value. Define $t_{n+1}$ to be the minimum time $t$ such that every mobile in $\mathcal{B}\left(t_{n+1}^{\prime}\right)$ has performed both power-up and power-down action after $t_{n+1}^{\prime}$, and every mobile $i$ in $\mathcal{A}\left(t_{n+1}^{\prime}\right)$ has moved its power at least half way (in log scale) from $P_{i}^{\left(t_{n+1}^{\prime}\right)}$ to its limiting value. If both $\mathcal{B}\left(t_{n+1}^{\prime}\right)$ and $\mathcal{A}\left(t_{n+1}^{\prime}\right)$ are empty sets, then $t_{n+1}:=t_{n+1}^{\prime}+1$. The idea can be roughly described by Fig. 5 .

Lemma 7: In a weakly canonical system, there exists a positive number $\underline{P}$ such that $P_{i}^{(t)}>\underline{P}$ for all $i \in \mathcal{A}\left(t_{1}\right) \cup \mathcal{B}\left(t_{1}\right)$ and $t>t_{1}$. 


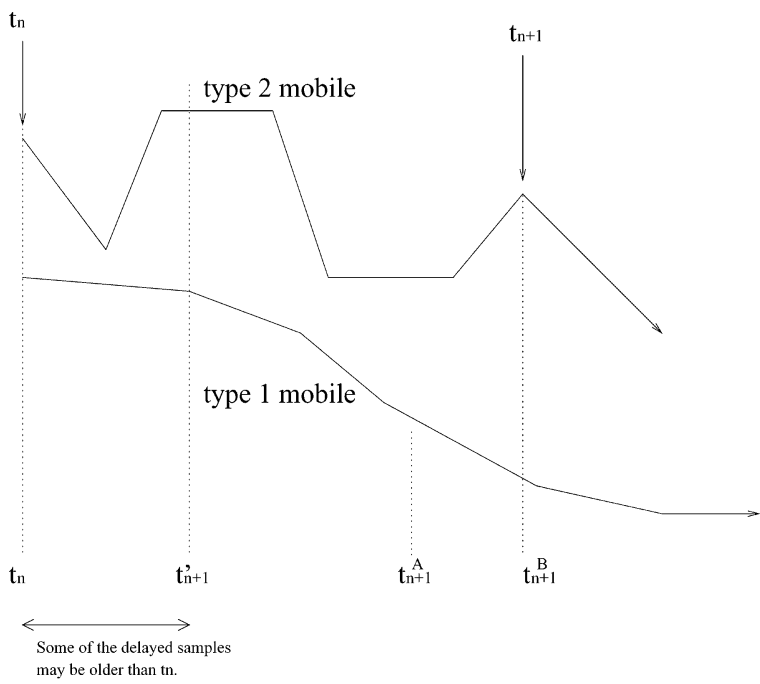

Fig. 5. Illustration of defining $t_{n+1}$ from $t_{n}$.

Proof: From the definition of $t_{1}, P_{i}^{\left(t_{1}\right)}>0$ for all $i \in$ $\mathcal{A}\left(t_{1}\right) \cup \mathcal{B}\left(t_{1}\right)$ and $t>t_{1}$. By using a similar argument as used in proving the bounded power theorem, with " $\leq$ " changed to " $\geq$ " and "max" changed to "min," etc., we prove that $P_{i}^{(t)}$ are all bounded below by a positive number for all $i \in \mathcal{A}\left(t_{1}\right) \cup \mathcal{B}\left(t_{1}\right)$ and $t>t_{1}$.

Lemma 8: Let $h_{i, \alpha}(\boldsymbol{P})=\alpha I_{i}(\boldsymbol{P})-I_{i}(\alpha \boldsymbol{P})$ for $\alpha>0$. Then, for any fixed $\boldsymbol{P}, \boldsymbol{h}_{i, \alpha}(\boldsymbol{P})$ is monotonically increasing in $\alpha$.

Proof: For any $\alpha \geq \delta>0$. We let $\alpha=\alpha^{\prime} \delta$ where $\alpha^{\prime} \geq 1$

$$
\begin{aligned}
h_{i, \alpha}(\boldsymbol{P}) & =\alpha^{\prime} \delta I_{i}(\boldsymbol{P})-I_{i}(\alpha \boldsymbol{P}) \\
& =\alpha^{\prime} h_{i, \delta}(\boldsymbol{P})+\alpha^{\prime} I_{i}(\delta \boldsymbol{P})-I_{i}\left(\alpha^{\prime}(\delta \boldsymbol{P})\right) \\
& \geq \alpha^{\prime} h_{i, \delta}(\boldsymbol{P}) .
\end{aligned}
$$

We define a measure of the power change for each mobile as follows. Given a positive integer $K$, for user $i$, define $\Delta_{i}(n, K)$ as

$$
\begin{aligned}
& \Delta_{i}(n, K):= \\
& \begin{cases}\max _{t_{n} \leq \zeta_{1} \leq \zeta_{2} \leq t_{K+1}}\left|\log \frac{P_{i}^{\left(\zeta_{1}\right)}}{P_{i}^{\left(\zeta_{2}\right)}}\right|, & \text { if } i \in \mathcal{A}\left(t_{n}\right) \cup \mathcal{B}\left(t_{n}\right) \\
0, & \text { if } i \in \mathcal{C}\left(t_{n}\right) .\end{cases}
\end{aligned}
$$

The bounded power theorem and Lemma 7 imply that the above logorithm is finite. Also, because the maximum in $\Delta_{i}(n, K)$ is over a finite period $\left(P_{i}^{\left(\zeta_{1}\right)}\right.$ and $P_{i}^{\left(\zeta_{2}\right)}$ take only finitely many different values), $\Delta_{i}(n, K)$ exists and is finite for $1 \leq n \leq K$. Then we define the power change of the whole system as

$$
D_{K}(n):=\max _{i} \Delta_{i}(n, K)
$$

for $1 \leq n \leq K$, and also define

$$
d_{K}(n):=D_{K}(n)-D_{K}(n+1)
$$

for $1 \leq n \leq K-1$. See Fig. 6 for an illustration of the power change of mobile 1 to illustrate the relation of $\zeta_{1}, \zeta_{2}, \zeta_{3}$, and $\zeta_{4}$.

Proposition 9: Given any $\bar{\delta}>0$, there exists a positive constant $\bar{\omega}$ such that

$$
D_{K}(n+1)>\bar{\delta} \text { implies } d_{K}(n)>\bar{\omega}
$$

for all $K \geq 2$ and $1 \leq n \leq K-1$.

Proof: Since the powers of the mobiles are bounded, let $\mathcal{P}=\left[0, \tilde{P}_{1}\right] \times \cdots \times\left[0, \tilde{P}_{N}\right]$, where $\tilde{P}_{i}$ is the upper bound of the power of user $i$, and $h_{i, \alpha}(\boldsymbol{P})=\alpha I_{i}(\boldsymbol{P})-I(\alpha \boldsymbol{P})$. Let $\bar{\delta}=\log \delta$ and $\bar{\omega}=\log \omega$. Let $I_{0}=\sup _{i, t} I_{i}\left(\boldsymbol{P}^{\left(\vec{\tau}_{i}(t)\right)}\right), \nu=$ $\lim _{K \rightarrow \infty} D_{K}(1)$ and $\epsilon(\alpha)=\min _{i} \min _{\boldsymbol{P} \in \mathcal{P}} h_{i, \alpha}(\boldsymbol{P})$. Note that $\epsilon(\alpha)$ is monotonically increasing in $\alpha$. The supremum in $I_{0}$ exists and is finite, since $\boldsymbol{P}^{\left(\vec{\tau}_{i}(t)\right)}$ is bounded and $I_{i}$ is continuous in $\boldsymbol{P}$ for all nonnegative $\boldsymbol{P} \cdot \lim _{K \rightarrow \infty} D_{K}(1)$ exists, since $D_{K}(1)$ is a bounded and monotonically increasing sequence in $K$. Minimum for $h_{i, \alpha}(\boldsymbol{P})$ exists, since $I_{i}(\boldsymbol{P})$ is uniformly scalable in $\mathcal{P}$. Also note that $\epsilon(\alpha)$ is an increasing function in $\alpha$. We will prove the proposition with $\omega=\min \left\{\delta, 1+\left(\epsilon(\delta) / \nu I_{0}\right)\right\}>1$.

Let $D_{K}(n+1)=\bar{\delta}^{\prime}>\bar{\delta}$. Without loss of generality, assume $\log \left(P_{1}^{\left(\zeta_{2}\right)} / P_{1}^{\left(\zeta_{4}\right)}\right)=\bar{\delta}^{\prime}$, where $t_{n+1} \leq \zeta_{2} \leq \zeta_{4} \leq t_{K+1}$. (The argument is similar for the case that $\log \left(P_{1}^{\left(\zeta_{2}\right)} / P_{1}^{\left(\zeta_{4}\right)}\right)=-\bar{\delta}^{\prime}$.) We then have

$$
P_{1}^{\left(\zeta_{2}\right)}=\delta^{\prime} P_{1}^{\left(\zeta_{4}\right)} .
$$

We also know that mobile 1 either belongs to $\mathcal{A}\left(t_{n}\right)$ or $\mathcal{B}\left(t_{n}\right)$. We divide the problem into two cases.

Case 1) $1 \in \mathcal{A}\left(t_{n+1}^{\prime}\right)$

Recall that $l_{1}$ is the limiting value of the power of user 1. Since the power of mobile 1 changes monotonically, we can show that $\log \left(P_{1}^{\left(t_{n+1}\right)} / l_{1}\right) \geq \bar{\delta}^{\prime}$. From the definition of $t_{n+1}$, we have $\log \left(P_{1}^{\left(t_{n+1}^{\prime}\right)} / P_{1}^{\left(t_{n+1}\right)}\right) \geq \bar{\delta}^{\prime}$. Together with $\log \left(P_{1}^{\left(t_{n+1}\right)} / P_{1}^{\left(\zeta_{4}\right)}\right) \geq \bar{\delta}^{\prime}$, we have $\log \left(P_{1}^{\left(t_{n+1}^{\prime}\right)} / P_{1}^{\left(\zeta_{4}\right)}\right) \geq 2 \bar{\delta}^{\prime}$. Since $t_{n} \leq t_{n+1}^{\prime} \leq$ $\zeta_{4} \leq t_{n+1}$, we have $D_{K}(n) \geq 2 \bar{\delta}^{\prime}$. Then $d_{K}(n)=D_{K}(n)-D_{K}(n+1) \geq \bar{\delta}^{\prime} \geq \bar{\omega}$ for this case.

Case 2) $1 \in \mathcal{B}\left(t_{n+1}^{\prime}\right)$

Let $\zeta_{1}$ be the time of the last power-up action before $\zeta_{2}$. By the bounding condition, $P_{1}^{\left(\zeta_{1}\right)} \leq P_{1}^{\left(\zeta_{1}+1\right)} \leq$ $\gamma_{1} I_{1}^{\left(\zeta_{1}\right)}$. Hence

$$
P_{1}^{\left(\zeta_{2}\right)} \leq P_{1}^{\left(\zeta_{1}+1\right)} \leq \gamma_{1} I_{1}^{\left(\zeta_{1}\right)} .
$$

The inequality follows since there is no power-up from time $\zeta_{1}+1$ to $\zeta_{2}$.

On the other hand, the power change of mobile 1 just before time $\zeta_{4}$ (at time $\left.\zeta_{3}\right)$ must be power-down. Otherwise, $D_{K}(n+$ 1) $>\bar{\delta}^{\prime}$, which contradicts the assumption that $D_{K}(n+1)=\bar{\delta}^{\prime}$. The bounding condition, $\gamma_{1} I_{1}^{\left(\zeta_{3}\right)} \leq P_{1}^{\left(\zeta_{4}\right)} \leq P_{1}^{\left(\zeta_{3}\right)}$, implies

$$
\begin{aligned}
& \gamma_{1} I_{1}^{\left(\zeta_{3}\right)} \leq P_{1}^{\left(\zeta_{2}\right)} / \delta^{\prime} \quad(\operatorname{using}(12)) \\
& \gamma_{1} I_{1}^{\left(\zeta_{3}\right)} \leq \gamma_{1} I_{1}^{\left(\zeta_{1}\right)} / \delta^{\prime} \quad(\operatorname{using}(13)) \\
& \delta^{\prime} I_{1}^{\left(\zeta_{3}\right)} \leq I_{1}^{\left(\zeta_{1}\right)} .
\end{aligned}
$$

Moreover, Theorem 4 implies that the powers of the users are bounded, so we may apply Lemma 3 and obtain

$$
\begin{aligned}
\delta^{\prime} I_{1} & \left(\boldsymbol{P}^{\left(\vec{\tau}_{1}\left(\zeta_{3}\right)\right)}\right)-I_{1}\left(\omega \delta^{\prime} \boldsymbol{P}^{\left(\vec{\tau}_{1}\left(\zeta_{3}\right)\right)}\right) \\
\geq & \delta^{\prime} I_{1}\left(\boldsymbol{P}^{\left(\vec{\tau}_{1}\left(\zeta_{3}\right)\right)}\right) \\
& -\left[\omega \delta^{\prime} I_{1}\left(\boldsymbol{P}^{\left(\vec{\tau}_{1}\left(\zeta_{3}\right)\right)}\right)-\epsilon\left(\delta^{\prime}\right)\right]
\end{aligned}
$$




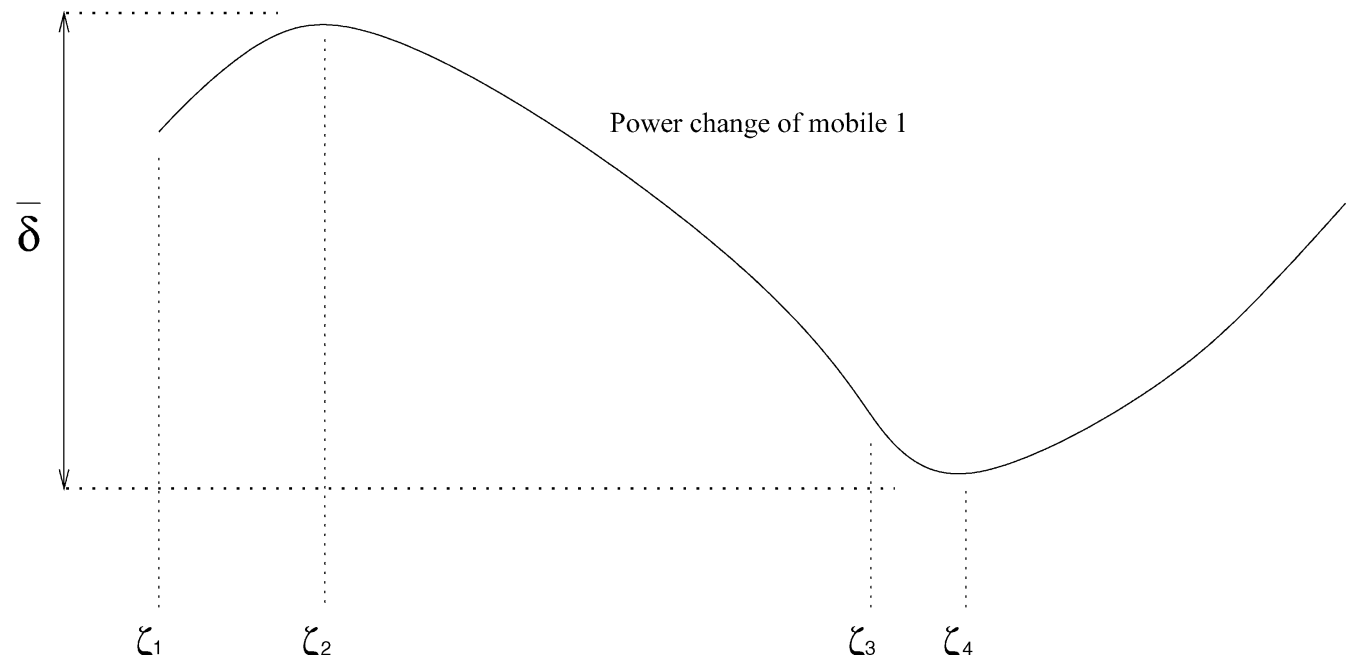

Fig. 6. Relation of $\zeta_{1}, \zeta_{2}, \zeta_{3}$, and $\zeta_{4}$.

$$
\begin{aligned}
= & \delta^{\prime} I_{1}\left(\boldsymbol{P}^{\left(\vec{\tau}_{1}\left(\zeta_{3}\right)\right)}\right)-\left[\delta^{\prime} I_{1}\left(\boldsymbol{P}^{\left(\vec{\tau}_{1}\left(\zeta_{3}\right)\right)}\right)\right. \\
& \left.+(\omega-1) \delta^{\prime} I_{1}\left(\boldsymbol{P}^{\left(\vec{\tau}_{1}\left(\zeta_{3}\right)\right)}\right)-\epsilon\left(\delta^{\prime}\right)\right] \\
\geq & \delta^{\prime} I_{1}\left(\boldsymbol{P}^{\left(\vec{\tau}_{1}\left(\zeta_{3}\right)\right)}\right)-\delta^{\prime} I_{1}\left(\boldsymbol{P}^{\left(\vec{\tau}_{1}\left(\zeta_{3}\right)\right)}\right) \\
& +\epsilon\left(\delta^{\prime}\right)-(\omega-1) \delta^{\prime} I_{0} \\
= & \epsilon\left(\delta^{\prime}\right)-(\omega-1) \delta^{\prime} I_{0} \\
\geq & \epsilon\left(\delta^{\prime}\right)-\epsilon(\delta) \geq 0 .
\end{aligned}
$$

Among those $(M+1) d_{K+1}(n)$ 's, at least one of them, say $d_{K+1}\left(n^{\prime}\right)$ where $1 \leq n^{\prime} \leq M+1$, is smaller than $\bar{\omega}$. By Proposition $9, D_{K+1}\left(n^{\prime}+1\right)<\bar{\delta}$. This implies that $D_{K+1}(M+2)<$ $\bar{\delta}$. Since $K$ is arbitrarily large, the power change of the mobiles will not exceed $\bar{\delta}$ after $t_{M+2}$. Since $\bar{\delta}$ is arbitrarily small, we conclude that power vector $\lim _{t \rightarrow \infty} \boldsymbol{P}^{(t)}$ exists, and therefore, $\lim _{t \rightarrow \infty} \Gamma^{(t)}$ also exists.

\section{ACKNOWLEDGMENT}

The last line follows since

$$
\omega=\min \left\{\delta, 1+\frac{\epsilon(\delta)}{\nu I_{0}}\right\} \leq 1+\frac{\epsilon(\delta)}{\delta I_{0}} .
$$

Combined with (16), we have

$$
I_{1}\left(\boldsymbol{P}^{\left(\vec{\tau}_{1}\left(\zeta_{1}\right)\right)}\right) \geq \delta^{\prime} I_{1}\left(\boldsymbol{P}^{\left(\vec{\tau}_{1}\left(\zeta_{3}\right)\right)}\right) \geq I_{1}\left(\omega \delta \boldsymbol{P}^{\left(\vec{\tau}_{1}\left(\zeta_{3}\right)\right)}\right) .
$$

Therefore, a mobile $j$ exists such that

$$
P_{j}^{\left(\tau_{1}^{j}\left(\zeta_{1}\right)\right)} \geq \omega \delta P_{j}^{\left(\tau_{1}^{j}\left(\zeta_{3}\right)\right)}
$$

for otherwise, the monotonicity of $I_{1}$ would be violated. Hence, we have

$$
\begin{aligned}
D_{K}(n) & \geq D_{K}(n+1)+\bar{\omega} \\
d_{K}(n) & \geq \bar{\omega} .
\end{aligned}
$$

\section{Proof of convergent power-control theorem}

Proof: By Lemmas 1 and $2, I_{i}(\boldsymbol{P})$ is positive and continuous. Consequently, $\Gamma_{i}(\boldsymbol{P})=\left(P_{i}\right) /\left(I_{i}(\boldsymbol{P})\right)$ is a continuous function of $\boldsymbol{P}$. Therefore, if $\lim _{t \rightarrow \infty} \boldsymbol{P}^{(t)}$ exists, $\lim _{t \rightarrow \infty} \boldsymbol{\Gamma}\left(\boldsymbol{P}^{(t)}\right)$ exists and equals $\boldsymbol{\Gamma}\left(\lim _{t \rightarrow \infty} \boldsymbol{P}^{(t)}\right)$.

We let $X=\sup _{i} D_{i}(1)$. By Theorem 4 and Lemma 7 , given any initial power vector $\boldsymbol{P}^{(0)}$, the range of the power change is bounded above and below by a positive number, hence, $X$ is finite. For any positive real number $\bar{\delta}$, we have the corresponding $\bar{\omega}$ given by Proposition 9. We let $M=\lceil X / \bar{\omega}\rceil$, where $\lceil x\rceil$ is the smallest integer greater than or equal to $x$. For any positive integer $K \geq M+2$

$$
\begin{aligned}
& d_{K+1}(1)+\cdots+d_{K+1}(M+1) \\
& \quad=D_{K+1}(1)-D_{K+1}(M+2) \\
& \quad \leq M \bar{\omega} .
\end{aligned}
$$

\section{REFERENCES}

[1] N. Bambos, S. C. Chen, and G. Pottie, "Radio link admission algorithms for wireless networks with power control and active link quality protection," in Proc. IEEE INFOCOM, 1995, pp. 97-104.

[2] D. P. Bertsekas and J. N. Tsitsiklis, Parallel and Distributed Computation. Englewood Cliffs, NJ: Prentice-Hall, 1989.

[3] G. J. Foschini and Z. Miljanic, "A simple distributed autonomous power control algorithm and its convergence," IEEE Trans. Veh. Technol., vol. 42, pp. 641-646, Nov. 1993.

[4] D. Goodman and N. Mandayam, "Power control for wireless data," IEEE Pers. Commun. Mag., vol. 7, pp. 48-54, Apr. 2000.

[5] S. A. Grandhi, R. Vijayan, and D. J. Goodman, "Distributed power control in cellular radio systems," IEEE Trans. Commun., vol. 42, pp. 226-228, 1994.

[6] S. A. Grandhi and J. Zander, "Constrained power control in cellular radio systems," in Proc. IEEE Vehicular Technology Conf., 1994, pp. 824-828.

[7] J. D. Herdtner and E. K. P. Chong, "Analysis of a class of distributed asynchronous power control algorithms for cellular wireless systems," IEEE J. Select. Areas Commun., vol. 48, pp. 436-446, Mar. 2000.

[8] R. Jantti and S. L. Kim, "Second-order power control with asymptotically fast convergence," IEEE J. Select. Areas Commun., vol. 18, pp. 447-457, Mar. 2000.

[9] K. K. Leung, "Power control in CDMA systems," Master's thesis, Chinese Univ. Hong Kong, Shatin, NT, Hong Kong, Nov. 1999.

[10] D. Mitra, "An asynchronous distributed algorithm for power control in cellular radio systems," in Proc. 4th Winlab Workshop, New Brunswick, NJ, 1993, pp. 249-257.

[11] G. F. Simmons, Introduction to Topology and Modern Analysis. New York: McGraw-Hill, 1963

[12] C. W. Sung, K. K. Leung, and W. S. Wong, "A quality-based fixed-step power control algorithm with adaptive target threshold," IEEE Trans. Veh. Technol., vol. 49, pp. 1430-1439, July 2000.

[13] C. W. Sung and W. S. Wong, "A distributed fixed-step power control algorithm with quantization and active link quality protection," IEEE Trans. Veh. Technol., vol. 48, pp. 553-562, Mar. 1999. 
[14] — , "The convergence of an asynchronous cooperative algorithm for distributed power control in cellular systems," IEEE Trans. Veh. Technol., vol. 48, pp. 563-570, Mar. 1999.

[15] — , "Performance of a cooperative algorithm for power control in cellular systems with a time-varying link gain matrix," ACM Wireless Network, vol. 6, no. 6, pp. 429-439, Dec. 2000.

[16] _ - "A noncooperative power control game for multirate CDMA data networks," in IEEE Trans. Wireless Commun., vol. 2, Jan. 2003, pp. $186-194$

[17] M. Xiao, N. B. Shroff, and E. K. P. Chong, "A utility-based powercontrol scheme in wireless cellular systems," IEEE/ACM Trans. Networking, vol. 11, pp. 210-221, Apr. 2003.

[18] R. D. Yates, "A framework for uplink power control in cellular radio systems," IEEE J. Select. Areas Commun., vol. 13, pp. 1341-1348, Sept. 1995.

[19] J. Zander, "Performance of optimum transmitter power control in cellular radio systems," IEEE Trans. Veh. Technol., vol. 41, pp. 57-62, Feb. 1992.

[20] J. Zander, "Distributed cochannel interference control in cellular radio systems," IEEE Trans. Veh. Technol., vol. 41, pp. 305-311, Aug. 1992.

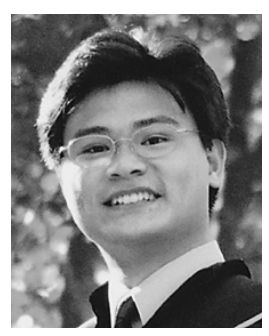

Kin Kwong Leung received the B.Eng. degree in 1997, the M.Phil. degree in 2000, and the Ph.D. degree in 2003, all from the Chinese University of Hong Kong, Shatin, NT, Hong Kong.

$\mathrm{He}$ is currently an Assistant Professor in the Department of Information Engineering, Chinese University of Hong Kong.

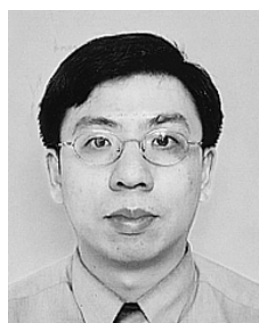

Chi Wan Sung (M'98) received the B.Eng., M.Phil., and $\mathrm{Ph} . \mathrm{D}$. degrees in information engineering from the Chinese University of Hong Kong, Shatin, NT, Hong Kong in 1993, 1995, and 1998, respectively.

After graduation, he was appointed Visiting Assistant Professor with the Department of Information Engineering, Chinese University of Hong Kong. He joined the City University of Hong Kong, Kowloon, Hong Kong, in 2000 and was an Assistant Professor in the Department of Computer Science. Since September 2001, he has been with the Department of Computer Engineering and Information Technology. His research interests include the area of wireless networks with an emphasis on power control, resource management, and signature sequence adaptation for CDMA systems.

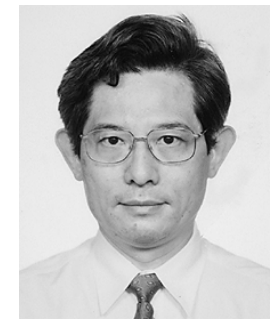

Wing Shing Wong (M'81-SM'90-F'02) received the combined M.A.B.A. degree in 1976 (summa cum laude), from Yale University, New Haven, CT. He received the M.S. degree in 1978 and the $\mathrm{Ph} . \mathrm{D}$. degree in 1980, both from Harvard University, Cambridge, MA

He joined AT\&T Bell Laboratories in 1982. From 1987 to 1992, he managed a group of technical staff working on a number of research and development projects and consulting activities. He joined the Chinese University of Hong Kong in 1992 and is now a Professor of Information Engineering. He was the Chairman of the Information Engineering Department from 1995 to 2003. Currently, he is serving as Science Advisor at the Innovation and Technology Commission of the HKSAR government. He is actively involved in a variety of $R \& D$ projects including topics such as mobile communication systems, search engine development, and information issues in estimation and control. He has published over 100 refereed journal and conference papers. He is a Visiting Professor of Southeast University, Nanjing.

Dr. Wong was an Associate Editor of the IEEE TRANSACTIONS ON AutOMATIC CONTROL for four years. He is the co-Editor-in-Chief of a new journal, Communications in Information and Systems. He is a Member of the 7th Council of the Chinese Association of Automation, and a Fellow of the Hong Kong Institution of Engineers.

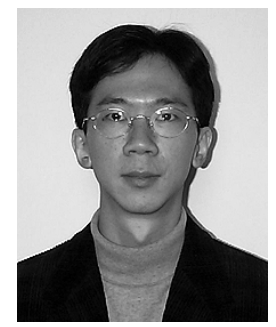

Tat M. Lok (S'95-M'95-SM'03) received the B.Sc. degree in electronic engineering from the Chinese University of Hong Kong, Shatin, NT, Hong Kong, in 1991, and the M.S.E.E. degree and the Ph.D. degree in electrical engineering from Purdue University, West Lafayette, IN, in 1992 and 1995, respectively.

He was a Postdoctoral Research Associate at Purdue University. In 1996, he joined the Chinese University of Hong Kong, where he is currently an Associate Professor. His research interests include CDMA systems, signal processing for communications, and communication theory. He has served in different positions for international conferences.

Dr. Lok cochaired the Wireless Access track of the IEEE Vehicular Technology Conference, Fall 2004. He is also serving as an Associate Editor for the IEEE TRANSACTIONS ON VEHICULAR TECHNOLOGY. 